\title{
Failure of penicillin prophylaxis in laboratory acquired leptospirosis
}

\author{
C.F. Gilks, ${ }^{1 *}$ H.P. Lambert, ${ }^{1}$ E.S. Broughton ${ }^{2}$ and C.C. Baker ${ }^{2}$ \\ ${ }^{1}$ St. George's Hospital, London SW17 ORE and ${ }^{2}$ Wellcome Research Laboratories, Kent, UK.
}

\begin{abstract}
Summary: A laboratory technician developed leptospirosis following accidential inoculation, despite prompt administration of parenteral penicillin by an accepted regimen of post-exposure prophylaxis. Another technician was similarly exposed and was given doxycycline; no illness or serological conversion followed. The implications of these cases are discussed and recommendations made for post-exposure chemoprophylaxis with doxycycline.
\end{abstract}

\section{Introduction}

Leptospirosis is one of the most widespread zoonoses, ${ }^{1}$ and human infection usually occurs through superficial cuts or abrasions after exposure to a contaminated environment, after contact with infected animals, or following rodent bites. ${ }^{2}$ Laboratory-associated infections are important and dangerous. ${ }^{3}$ Post-exposure prophylaxis is often required following accidental exposure to environments suspected as contaminated, after contact with, or bites from, infected animals, and following laboratory accidents. Penicillin given parenterally has been the recommended antibiotic ${ }^{4,5}$ but little information exists as to its efficacy; oral penicillin has failed. ${ }^{6}$ In pre-exposure prophylaxis doxycycline has been shown to have an efficacy of $95 \% .{ }^{7}$ We report the development of leptospirosis after a prolonged incubation period following a laboratory accident, despite the prompt administration of parenteral penicillin.

\section{Case reports}

\section{Case 1}

A 23 year old technician working in a microbiological research laboratory dropped a flask of Leptospira interrogans serogroup Icterohaemorrhagiae (strain CF1); she put on gloves and cleaned the spillage. One hour after removing the

Correspondence: Professor H.P. Lambert, M.D., F.R.C.P. *Present address: Nuffield Department of Medicine, John Radcliffe Hospital, Oxford, UK.

Accepted: 20 October 1987 gloves she noted a cut on one finger, but no blood was seen. She was given an intramuscular injection of Triplopen (benethamine penicillin $475 \mathrm{mg}$; procaine penicillin $250 \mathrm{mg}$, sodium benzyl penicillin $300 \mathrm{mg}$ ); this dose was repeated three days later. She continued at work and was not involved in any other spills. She had no further cuts or abrasions which, by the rules of the laboratory, would have disbarred her from working with this pathogen. She had minimal contact with infected animals when she wore protective clothing including gloves and visor; she was not bitten.

Forty days after the accident, she became unwell with headache, myalgia, pleural pain and symmetrically painful but not swollen joints. She had remittent fever up to $39.4^{\circ} \mathrm{C}$ with sweating and occasional rigors over 9 days. On the ninth day of illness motile leptospires were identified on darkfield examination of serum from a freshly clotted sample of blood and dark field examination of a fresh urine sample showed a few non-motile forms suggestive of leptospires.

She received a further intramuscular dose of benzylpenicillin $(600 \mathrm{mg})$, and after this was afebrile but remained unwell with mildly abnormal liver function tests. She was therefore admitted to hospital and given intravenous benzylpenicillin $1.2 \mathrm{~g}$ six hourly for 5 days. On discharge 59 days after infection she was well.

Three days before the accident she was serologically negative (less than 1 in 2 by the microscopic agglutination test, MAT) to a range of leptospiral antigens including Icterohaemorrhagiae. Ten days after the start of symptoms and 50 days from presumed infection she was strongly positive

(C) The Fellowship of Postgraduate Medicine, 1988 
by leptospiral macroscopic slide agglutination, had a complement fixation titre of 1 in 160 and a MAT titre of 1 in 320 to serogroups Icterohaemorrhagiae and Canicola. Three months later titres had declined, with a maximum titre of 1 in 640 (MAT) to Icterohaemorrhagiae.

\section{Case 2}

A healthy 35 year old technician working in the same establishment stabbed her thumb with a sterile scalpel blade through gloves contaminated with fresh blood from an animal infected with $L$. interrogans serogroup Sejroe serovar hardjo. The wound was cleaned and dressed, and she was started that day on $100 \mathrm{mg}$ doxycycline which was continued twice weekly for 6 weeks. She remained in good health following exposure. Agglutinating antibody to Leptospira interrogans serovar hardjo remained negative with titres less than 1 in 2 sixty days after exposure.

\section{Discussion}

Leptospirosis is one of the most widespread zoonoses $^{1}$ and infection is usually related to occupational exposure, primarily from direct or indirect contact with urine of infected animals ${ }^{5}$ or bites from infected animals. ${ }^{2}$

Laboratory staff are among the groups at risk, both from working with the pathogen itself or through handling infected animals. $\mathrm{Pike}^{3}$ in a comprehensive compilation of 3921 cases of laboratory-associated infections of all causes between 1930 and 1974 recorded 67 cases of leptospirosis $-1.7 \%$ overall. It was the fifteenth most prevalent laboratory-associated pathogen and was responsible for 10 out of 167 deaths $(6.1 \%)$. Collins $^{8}$ lists 25 papers worldwide, most recently in 1974.

Several common laboratory animals are sources of risk. Dogs and rodents are the natural reservoirs of several serovars of pathogenic leptospires; infection or carriage is prolonged and often inapparent. Mice are a common natural host $\mathbf{9}^{910}$ and one laboratory, ${ }^{9}$ in a retrospective investigation of 58 people who handled white mice found that 8 had serological evidence of previous infections; 6 of these recalled severe undiagnosed febrile illnesses. Direct handling of the pathogen has also resulted in infection. A case of leptospirosis following needle prick was reported by Welcker ${ }^{11}$ in 1938 , before penicillin prophylaxis was possible. We are also aware of a similar, unpublished, canicola infection diagnosed retrospectively, in which the inoculum was approximately ten organisms.
Broom and Norris ${ }^{6}$ reported accidental selfinoculation by a technician with leptospires of serogroup Javanica. Despite wound toilet and immediate administration of oral penicillin 1 megaunit (preparation not specified), followed by 0.5 megaunits 6 hourly for three days, clinical illness developed after 10 days when leptospires were seen in blood and successfully cultured. The incubation period was thus not modified. In our first case of accidental inoculation followed by parenteral penicillin, leptospirosis developed although the recognized incubation period of 7-20 days, commonly 7-14,4,5 was exceeded by 20 days; it is impossible to say whether the severity of eventual illness was modified by post-exposure prophylaxis. This observation provides another example of a well documented paradox in the pathogenesis of infection, which has also been observed in scrub typhus ${ }^{12}$ and in typhoid, ${ }^{13}$ that an apparently logical scheme of post-exposure prophylaxis may not in practice prevent illness but merely prolong incubation.

What then should be recommended for prophylaxis following exposure to leptospires? The standard recommended regimen, parenteral penicillin 1.2 to $2.4 \mathrm{~g} /$ day for 5 days, ${ }^{4,5}$ is based on the treatment of established disease rather than on any direct evidence of efficacy in prophylaxis. Oral penicillin has failed ${ }^{6}$ and as we report, parenteral long acting penicillins may not work. A more prolonged course of benzylpenicillin might well be effective but the problems of administration and acceptability for a well person at work would be much increased especially if the intravenous route is used.

Of the possible methods of prophylaxis using oral administration, erythromycin or a tetracyline, $1 \mathrm{~g} /$ day for 5 days, has been suggested as an alternative to penicillin. ${ }^{5}$ Amoxycillin has strong leptospiricidal activity in vitro ${ }^{14}$ and has been used in treatment with apparent success..2.15 Experience of these antibiotics for post-exposure prophylaxis is however limited, and short courses cannot now be recommended because of the possibility that disease will develop after a prolonged incubation period.

Doxycycline has been shown to be an excellent true (pre-exposure) prophylactic agent in a prospective randomized double-blind trial in 940 troops who spent three weeks of jungle training in Panama. ${ }^{7}$ The drug was given in a dose of $200 \mathrm{mg}$ pre-exposure on arrival in Panama, then weekly and at the end of exposure on completing training. 20 leptospira infections occurred in the placebo group $(4.2 \%)$ compared with one in the doxycycline group $(0.2 \%)$ indicating a protective efficiency of $95 \%$. It is interesting to note that doxycycline is also effective in the prophylaxis of scrub typhus. ${ }^{16}$ 
This study may not be directly applicable for postexposure treatment, when the leptospires may have several hours in the host before effective drug levels are reached. Moreover, in laboratory accidents the route of infection and the dose may also be different. Nevertheless doxycycline is a potent leptospiricidal antibiotic and can be given for an extended period of time in a simple oral regimen.

We consider that doxycycline $100 \mathrm{mg}$ twice weekly over a period of 6 weeks provides a rational regimen for post-exposure prophylaxis, which takes into account the possibility of prolonged incubation. The second accidental exposure we describe was treated in this way. Neither illness nor seroconversion ensued, although we cannot of course know whether infection would have occurred in the

\section{References}

1. Feigin, R.D. \& Anderson, D.C. Human leptospirosis. CRC Crit Rev Clin Lab Sci 1975, 5: 413-467.

2. Waitkins, R.A. Update on leptospirosis. $\mathrm{Br}$ Med $J$ 1985, 290: 1502-1503.

3. Pike, R.M. Laboratory associated infections. Summary and analysis of 3291 cases. Health Lab Sci 1976, 13: 105-114.

4. Turner, L.H. Leptospirosis. Trans $R$ Soc Trop Med Hyg 1967, 61: 842-854.

5. Faine, S. (ed) Guidelines for the Control of Leptospirosis. WHO Offset Publication, 1982, No. 67, WHO, Geneva.

6. Broom, J.B. \& Norris, T.St.M. Failure of prophylactic penicillin to inhibit a human laboratory case of leptospirosis. Lancet 1957, i: 721-722.

7. Takafuji, E.T., Kirkpatrick, J.W., Miller, R.N. et al. An efficacy trial of doxycycline chemoprophylaxis against leptospirosis. $N$ Engl J Med 1984, 310: 497500 .

8. Collins, C.H. Laboratory-acquired Infections. Butterworths, London, 1983.

9. Barkin, R.M., Guckian, J.C. \& Gosser, J.W. Infection by Leptospira ballum: a laboratory acquired associated case. South Med J 1974, 67: 155.

10. Stoenner, H.G. \& Maclean, D. Leptospirosis (Ballum), contracted from Swiss Albino mice. Arch Int Med 1958, 101: 606-610. absence of chemoprophylaxis. This regimen provides a valid and more acceptable alternative to the short course parenteral regimens previously recommended and is simpler to comply with over a prolonged course than amoxycillin. Any febrile illness which develops in the two or three month period following exposure should be investigated as a possible breakdown in prophylaxis.

\section{Acknowledgements}

We thank the staff of the Leptospira Reference Unit, Public Health Laboratory Service (Director Dr S.A Waitkins) for performing the serological examinations.

11. Welcker, A. Laboratoriums infektionen mit Weilscher Krakheit. Zbl Bakt abl Orig 1934, 141, 400-410. Quoted in Collins (Reference 8).

12. Smadel, J.E., Traub, R., Frick, L.P., Diercks, F.H. \& Bailey, C.A. Chloramphenicol in the chemoprophylaxis of scrub typhus (tsutsugamusohi disease) III. Suppression of overt disease by prophylactic regimens of four weeks duration. Am J Hyg 1950, 51: 216-228.

13. Hornick, R.B., Greisman, S.E., Woodward, T.G. Dupont, H.L., Dawkins, A.T. \& Synder, M.J Typhoid fever, pathogenesis and immunologicas control. N Engl J Med 1970, 283: 686-691.

14. Broughton, E.S. \& Slack, L.E. The susceptibility of a strain of Leptospira interrogans serogroup Icterohaemorrhagiae to amoxycillin, erythromycin, lincomycin, tetracycline, oxytetracycline and minocycline. Zbl Bakt Hyg A 1986, 261: 425-431.

15. Munnich, D. \& Lakatos, M. Treatment of human leptospira infections with semicillin (ampicillin) or amoxil (amoxycillin). Chemotherapy 1976, 22: 372380.

16. Twartz, J.L., Shirai, A., Servaraju, G., Saunders, J.P., Huksoll, D.L. \& Groves, M.G. Doxycycline prophylaxis for human scrub typhus. J Infect Dis 1982, 146: 811-818. 